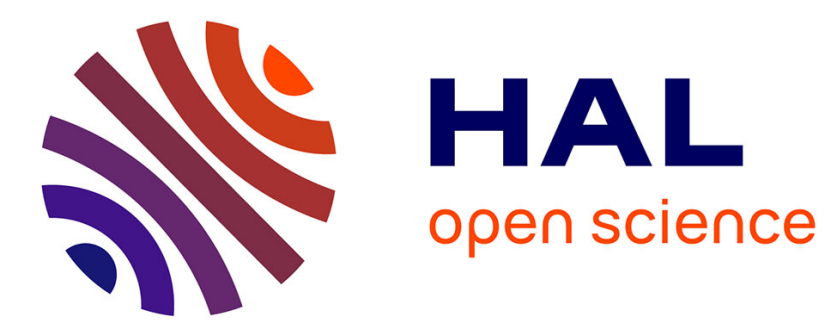

\title{
Sociologie de la formation post-scolaire
}

Claude Dubar, Charles Gadea, Coralie Perez

\section{To cite this version:}

Claude Dubar, Charles Gadea, Coralie Perez. Sociologie de la formation post-scolaire. P. Carré et P. Caspar. Traité des sciences et des techniques de la formation, DUNOD, pp.125-142, 2011. halshs00646423

\section{HAL Id: halshs-00646423 \\ https://shs.hal.science/halshs-00646423}

Submitted on 2 Apr 2021

HAL is a multi-disciplinary open access archive for the deposit and dissemination of scientific research documents, whether they are published or not. The documents may come from teaching and research institutions in France or abroad, or from public or private research centers.
L'archive ouverte pluridisciplinaire HAL, est destinée au dépôt et à la diffusion de documents scientifiques de niveau recherche, publiés ou non, émanant des établissements d'enseignement et de recherche français ou étrangers, des laboratoires publics ou privés. 


\section{Sociologie de la formation post-scolaire ${ }^{1}$}

\section{Introduction}

La sociologie de la formation n'a rien d'une branche récente ou mineure de la sociologie. Elle s'appuie sur un fonds d'interrogations et d'analyses initiées dès les années cinquante-soixante, et la place qu'elle occupe parmi les centres d'intérêt des chercheurs n'a cessé depuis de s'accroître, au point de se voir parfois érigée en spécialité rivale de la sociologie de l'éducation ${ }^{2}$. Pourtant, elle demeure un ensemble complexe et mal unifié. On peut songer à un carrefour où se rencontrent des lignes diverses de recherche, venant les unes de la sociologie de l'éducation, les autres de la sociologie du travail et des organisations, mais aussi de la psychologie sociale, de l'histoire et de l'économie. Un relatif consensus la situe au cœur d'un faisceau de travaux gravitant autour du thème de la relation entre la formation et l'emploi, une relation qu'on s'accorde parfois à considérer comme « introuvable ${ }^{3} »$.

Sans doute une part de la difficulté à définir son domaine tient-elle aux ambiguïtés du vocabulaire. Des termes tels que formation, formation professionnelle initiale ou continue, formation ou éducation permanente, auxquels s'ajoute la " formation tout au long de la vie » (lifelong learning), lancée par la Communauté européenne en 1996, s'inscrivent dans une histoire tissée d'entrelacs et d'oppositions subtiles qui requièrent de longs ouvrages pour en préciser le sens. Cette confusion ne dure-t-elle que par manque de conceptualisation ? C'est en partie vrai, tant les schèmes de pensée les plus divers s'accumulent sans qu'une perspective sociologique d'ensemble parvienne à s'imposer et ordonner le paysage théorique. Une tâche aussi vaste déborde le propos de cette contribution. Nous nous contenterons donc ici de préciser rapidement en quel sens nous parlons de formation post-scolaire.

Avec C. Maroy, nous partirons de l'idée que la formation est dite professionnelle lorsqu'elle « définit son public cible ou son contenu en référence aux activités de travail ${ }^{4}$. Elle peut être considérée comme continue si, d'un point de vue pédagogique, elle s'appuie ou prolonge une première formation initiale. Mais, dans un sens plus large, toute formation s'adressant à des personnes insérées dans la vie active, qu'elles soient en emploi ou au chômagerelève de la formation continue. Puisqu'elle s'adresse alors à des adultes qui ont quitté le système scolaire, la formation continue peut être qualifiée de post-scolaire et ces deux termes deviennent interchangeables. Contrairement à l'expression formation permanente, terme un peu flou qui

\footnotetext{
${ }^{1}$ Par Claude Dubar, Charles Gadea et Coralie Perez.

${ }^{2}$ L. Tanguy (1998). « La formation, une activité sociale en voie de définition », in De Coster et Pichault (éds.), Traité de sociologie du travail, Bruxelles, De Boeck Université, 2 édition, p. 185-212.

${ }^{3}$ L. Tanguy (1986). «Introduction » in L. Tanguy (dir.), L'introuvable relation formationemploi, Paris, La Documentation française, p. 7-34.

${ }^{4}$ C. Maroy (1998). « La formation en entreprise : de la gestion de la main-d'œuvre à l'organisation qualifiante $»$, in De Coster et Pichault, éds., Traité de sociologie du travail, Bruxelles, De Boeck Université, $2^{\mathrm{e}}$ édition, p. 297.
} 
désigne en même temps un idéal éducatif global reliant formation initiale et éducation des adultes et des pratiques individuelles de formation très variées, souvent à dominante culturelle, le terme formation post-scolaire renvoie à des dispositifs de formation continue, dont les contours ont été progressivement tracés, en France, par une série de dispositions légales, en particulier par la loi de $1971^{5}$.

Tout comme les pratiques et institutions concernées, les objets de recherche regroupés sous ce terme forment un assemblage hétéroclite à cause du flottement de la terminologie et des différences de points de vue des sociologues. Ceux-ci engendrent des découpages différents du champ de la formation post-scolaire, à l'intersection de ceux du travail, de l'éducation et de la mobilité sociale et professionnelle. Dans ce traité, nous tenterons de donner un aperçu, nécessairement limité et partial, des approches sociologiques que nous jugeons les plus représentatives, en organisant l'exposé autour de quatre grandes dimensions. L'analyse des politiques et des dispositifs de formation précédera celle des pratiques et rapports sociaux de formation, qui débouchera sur l'étude des publics de la formation et des inégalités qui les traversent. La question des comparaisons internationales sera évoquée en conclusion.

Nous adopterons, pour chaque partie, une démarche diachronique en nous efforçant de retracer les grands axes des dynamiques de recherche concernant chacune de ces dimensions. Nous essaierons d'accorder une place importante aux recherches et données empiriques qui ont eu un impact significatif sur ces dynamiques.

\section{Approches sociologiques des politiques et dispositifs de formation}

Sans qu'il soit nécessaire de remonter au programme ambitieux tracé par Condorcet, dans son célèbre rapport auprès de l'Assemblée législative de 1791, on peut relever une série d'étapes et de glissements dans la manière dont les pouvoirs publics ont conçu et tenté de promouvoir la formation post-scolaire en France. Ces transformations ont donné lieu à quelques travaux de synthèse socio-historique ${ }^{6}$, mais surtout à de nombreuses observations, monographies et analyses de dispositifs ou institutions publics, dans des perspectives très variées. Quant aux politiques de formation des entreprises, elles ont retenu l'attention des sociologues depuis longtemps et ont alimenté une riche littérature.

\subsection{Les politiques publiques en France depuis 1959}

Même si elle a été précédée par des projets de loi restés sans suite, et par des initiatives locales impliquant des universités ou des centres associés du CNAM, la loi du 31 juillet 1959 relative à la "promotion sociale » marque le début d'une véritable prise en charge législative

${ }^{5}$ C. Dubar (2004). La formation professionnelle continue, Paris, La Découverte, coll. « Repères », $5^{\mathrm{e}}$ édition.

${ }^{6}$ N. Terrot (1997). Histoire de l'éducation des adultes en France. La part de l'éducation des adultes dans la formation des travailleurs, Paris, L'Harmattan ; Y. Palazzeschi (1998).

Introduction à une sociologie de la formation (1944-1994), anthologie de textes français, 2 volumes, Paris, L'Harmattan; C. Dubar, C. Gadéa (éds.) (1999). La promotion sociale en France, Lille, Presses Universitaires du Septentrion. 
de la question de la formation post-scolaire en France. Dans un pays marqué par les pénuries de main-d'œuvre de l'après-guerre et par de profondes divisions sociales et politiques, le gouvernement gaulliste de cette période se fixe un double objectif : favoriser la promotion du travail pour lutter contre les inégalités sociales et élever le niveau de formation et de qualification des salariés. Il faut bien garder à l'esprit que si les visées économiques ne sont pas absentes, elles ne constituent pas l'argumentation centrale du projet d'alors.

C'est par la suite que cette dimension prendra une place croissante, jusqu'à faire disparaitre toute référence à la promotion sociale, au profit de la seule formation professionnelle ${ }^{7}$. Ainsi, la fameuse «loi Delors » de juillet 1971 prolonge par certains aspects la « loi Debré » de 1959, la formation étant affichée comme un point-clé du dialogue social qui doit concilier les intérêts des entreprises et des salariés. Mais elle consacre aussi un retournement amorcé dès les lois de 1966 et 1968 : c'est le développement économique, et plus particulièrement la régulation de l'emploi, qui concentrent les priorités de l'État. La promotion sociale passe au second plan.

Cette loi introduit une distinction, qui sera confirmée en juillet 1978, entre les opérations de formation prévues dans les plans de formation des entreprises (de plus de 50 salariés) et financées par une contribution obligatoire des employeurs et le congé individuel de formation, relevant de l'initiative individuelle des salariés, financé par des organismes mutualisateurs. La logique économique prime dans l'orientation des formations «à l'initiative de l'employeur » (stages courts visant à accroître la compétitivité) qui forment la grande masse des opérations de formation, alors que les formations suivies « à l'initiative des salariés », notamment dans le cadre du Congé individuel de formation (CIF), concernent des effectifs beaucoup plus réduits, débouchent sur des formations longues, diplômantes, et relèvent encore d'une logique de promotion sociale qui, dès la fin des années soixante-dix, a du mal à trouver ses financements et à s'articuler avec la logique précédente.

La loi de 1984, dite « loi Rigout», puis la loi quinquennale (décembre 1993) font de la formation un moyen de réponse à la crise de l'emploi et d'adaptation aux mutations technologiques. Ce glissement conduisant à affecter les fonds publics aux dispositifs d'insertion des jeunes et de formation pour les chômeurs ne laisse plus qu'une place résiduelle aux formations promotionnelles. Désormais, les impératifs économiques dominent le champ de la formation devenue instrument du maintien de l'employabilité des salariés. Elle doit viser l'acquisition des « compétences recherchées par les entreprises » et celle-ci est de plus en plus individualisée. La formule de l'Union européenne « la formation tout au long de la vie », ne fait que renforcer, à partir de 1996, cette logique : chaque individu est appelé à devenir l'entrepreneur de sa propre formation continue.

L'accord national inter-professionnel signé en septembre 2003 par l'ensemble des organisations syndicales, patronales et salariées, repris par la loi du 4 mai 2004 intitulée "Formation tout au long de la vie professionnelle et le dialogue social », consacre cette logique visant à « permettre à chaque salarié d'être acteur de sa formation ». Dispositif phare de cette réforme du système institutionnel de formation continue, le Droit Individuel à la

${ }^{7}$ C. Dubar, C. Gadéa (éds.) (1999). La promotion sociale en France, op. cit. 
Formation $(\mathrm{DIF})^{8}$ devait apporter des réponses à deux enjeux : réduire les inégalités d'accès à la formation et permettre aux salariés de se prémunir contre les aléas de leur carrière professionnelle. Un premier jalon avait été posé avec la Validation des Acquis de l'Expérience (VAE) en 2002 (dans le cadre de la loi de «modernisation sociale ») qui autorise l'attribution de la totalité d'un titre ou d'un diplôme sur la base de l'expérience acquise dans une activité salarié ou non, et/ou bénévole. Avec la loi du 4 mai 2004, sont promues l'information et l'orientation professionnelle des salariés au travers de l'entretien professionnel, du bilan de compétences ou encore du passeport formation.

Les travaux préparatoires à cette réforme avaient rappelé la persistance de fortes disparités d'accès aux formations financées par l'employeur, au détriment des salariés les moins qualifiés et de ceux travaillant dans les petites et moyennes entreprises. L'écart croissant entre les formations financées par l'entreprise et celles suivies au titre du CIF, dont les effectifs stagnent (environ 20000 par an) et dont les acquis restent peu reconnus par l'entreprise, a rendue nécessaire la création d'une voie intermédiaire. Plusieurs tentatives visant la coconstruction, par l'employeur et le salarié, d'un projet de formation individualisé ont ainsi vu le jour, telles que l'accord interprofessionnel sur le co-investissement en 1991 ou le Capital Temps Formation en 1993. Toutefois, elles ont échoué faute de soutien et de consensus des organisations syndicales représentatives des salariés.

La réforme est engagée dans un contexte marqué par une convergence d'intérêts politiques et syndicaux pour créer une «assurance-emploi » ou "une sécurité sociale professionnelle ». Les débats autour de la réforme du système se sont nourris de propositions consistant à désencastrer la formation de l'emploi pour la rattacher, au moins en partie, à l'individu : droits de tirage sociaux ${ }^{9}$, droit individuel transférable et garanti collectivement ${ }^{10}$, passeport formation $^{11}$. Les partenaires sociaux ont fini par s'entendre sur le DIF, un compte individuel de formation abondé par l'entreprise mais " activé » à l'initiative du salarié avec l'accord de son employeur. Bien que novateur, le DIF ne fournit pas une réponse suffisante à tous les objectifs de la réforme. Il se voit en particulier reprocher de ne pas être transférable en cas de mobilité professionnelle.

Plusieurs rapports critiques sur le système institutionnel de formation ont présenté le DIF comme au milieu du gué, certains plaidant pour sa suppression ${ }^{12}$, d'autres recommandant au contraire de renforcer sa portabilité et d'étoffer les services d'information et d'orientation qui

\footnotetext{
${ }^{8}$ Le « Droit Individuel à la Formation » (DIF) octroie 20 heures de formation par an, cumulables sur six années (soit 120 heures) à tous les salariés en contrat à durée indéterminée à partir un an d'ancienneté dans l'entreprise. Les droits sont calculés prorata temporis pour les salariés travaillant à temps partiel.

${ }_{9}^{9}$ A. Supiot (1999), Au-delà de l'emploi. Transformations du travail et devenir du droit du travail en Europe, Flammarion.

${ }^{10}$ N. Pery (1999), La formation professionnelle : diagnostic, défis, enjeux. Contribution du Secrétariat aux droits des femmes et à la formation professionnelle, mars, La Documentation française.

${ }^{11} \mathrm{G}$. Lindeperg, (2000), Les acteurs de la formation professionnelle : pour une nouvelle donne. Rapport au Premier Ministre. Décembre, La Documentation française.

${ }^{12} \mathrm{P}$. Cahuc et A. Zylberberg, (2006), La formation professionnelle des adultes: un système à la dérive, Rapport pour le Centre d'Observation Economique de la CCIP, septembre.
} 
l'accompagnent ${ }^{13}$. C'est cette seconde voie qui a été reprise par les partenaires sociaux dans les accords nationaux interprofessionnels de 2008 et 2009.

Ainsi, la portabilité du DIF a été étendue : en cas de mobilité externe, le salarié peut, à son initiative, solder le nombre d'heures acquises au titre du DIF pour suivre une formation (ou un bilan de compétences, une VAE) chez son nouvel employeur, dans les deux années qui suivent son embauche ${ }^{14}$. Cette limite est en partie compensée par la constitution d'un « fonds paritaire de sécurisation des parcours professionnels » et abondé par une partie des contributions obligatoires des entreprises à la formation. Créé en 2009, ce fonds est destiné à financer l'accès à la qualification et à la requalification de salariés et de demandeurs d'emploi ciblés. Enfin, le « droit à l'information, l'orientation et la qualification professionnelles » est réaffirmé par la loi du 24 novembre 2009. Elle crée un «service dématérialisé gratuit et accessible à toute personne » de conseil et d'orientation professionnelle. Faut-il voir dans ces dernières législations un retour partiel vers l'esprit de l'éducation permanente (VAE, DIF) et de la promotion sociale ? Cela reste à vérifier car l'avenir et la portée de ces réformes restent encore incertains ${ }^{15}$.

\subsection{Les politiques de formation en entreprise}

L'intérêt porté par P. Naville aux questions de formation professionnelle ${ }^{16}$ et les travaux de G. Friedman ${ }^{17}$ sur les effets du changement technique sur l'apprentissage et la qualification ouvrière ont contribué à sensibiliser les sociologues français du travail aux enjeux de la formation en entreprise. Les premières recherches empiriques comparant les politiques de formation dans les grandes entreprises sont réalisés, dès la fin des années cinquante, souvent dans une perspective d'amélioration de la gestion du personnel ${ }^{18}$. Elles font ressortir une diversification des politiques de formation, entre l'objectif de permettre simplement une adaptation des salariés à leur travail et une intégration plus poussée à la gestion des ressurces humaines.. L'accès des salariés à la formation apparaît dans ces recherches comme lié aux changements techniques et économiques, ainsi qu'aux politiques de gestion du personnel et au thème souvent évoqué de la promotion sociale ${ }^{19}$.

$\mathrm{Au}$ cours de la période qui suit, des recherches s'inspirant des théories de la segmentation du marché du travail, considèrent prioritairement la formation comme un outil de gestion de la main-d'œuvre, dont les caractéristiques et les résultats varient en fonction de facteurs

${ }^{13}$ J.-C. Carle, (2007), Rapport sénatorial de la mission d'information sur le fonctionnement des dispositifs de formation professionnelle ; P. Ferracci, (2008), rapport du groupe de travail sur la réforme de la formation professionnelle.

${ }^{14}$ Mais le DIF n'est toujours pas «transférable »; le salarié peut solder ses droits acquis préalablement mais ne peut les transférer en cas de mobilité professionnelle.

${ }^{15}$ C. Dubar (2008), « Les changements possibles du système de formation continue », Formation Emploi, n 101, p.167-182.

${ }^{16} \mathrm{P}$. Naville (1948). La formation professionnelle et l'emploi, Paris, Marcel Rivière.

${ }^{17}$ G. Friedman (1947). Problèmes humains du machinisme industriel, Paris, Gallimard.

${ }^{18} \mathrm{R}$. Vatier (1960). Développement de l'entreprise et promotion des hommes, Paris, Éd. de 1'Entreprise moderne.

${ }^{19}$ C. et M. Durand (1971). De l'OS à l'ingénieur. Carrière et classes sociales, Paris, Éditions Ouvrières. 
structurels tels que la taille, la branche, l'intensité capitalistique et, plus particulièrement, le degré de développement du marché interne du travail ${ }^{20}$. Cette perspective insiste sur les rapports de classe dans l'entreprise et tend à minimiser la diversité des pratiques de formation, $\mathrm{y}$ compris dans les secteurs à forte concentration en capital ${ }^{21}$.

D'autres travaux, liés au courant de l'analyse stratégique, se centrent sur le système organisationnel de l'entreprise. Ils constatent que la formation, en redistribuant les formes de savoir ou d'expertise, fait naître de nouvelles relations entre les acteurs de l'entreprise qui s'expriment par un réagencement des réseaux d'alliances et par de nouvelles formes de pouvoir ou d'autorité..

La relation établie par Sainsaulieu ${ }^{22}$ entre «logiques de formation» et «logique de production » met en lumière des types d'acteurs différenciés qui renvoient à des formes contrastées d'identités au travail. Cette place essentielle que tient l'expérience de formation dans les dynamiques identitaires des adultes se verra confirmée par les travaux menés au cours des années quatre-vingt par un collectif de chercheurs qui s'intéressent aux innovations de formation dans les grandes entreprises et qui montreront que l'un des enjeux majeurs de ces politiques de formation réside dans la construction et la reconnaissance de formes spécifiques d'identité professionnelle chez les salariés.

En prise avec la montée des questions liées à la compétitivité des entreprises, la formation continue se transforme en profondeur, avec les changements des politiques de gestion des ressources humaines, mais aussi de l'organisation et des contenus du travail. Elle s'intègre aux stratégies de l'entreprise et aux changements du travail. Même si les sociologues concluent, en comparant de nombreux cas, à une " construction inachevée ${ }^{23}$ ", le paysage des formations en entreprise, depuis les années quatre-vingt-dix, n'a plus grand chose à voir avec celui issu de la législation de 1971

Un tableau d'ensemble est fourni par la série de monographies d'entreprises de taille et de secteur différents qui s'est attachée en 2004 à analyser la diversité des politiques de formation en croisant le regard des différents acteurs (salariés, encadrement, responsable de formation, représentants du personnels) ${ }^{24}$. Elle montre notamment que les entreprises engagées dans une démarche compétence et faisant de l'entretien individuel le point nodal de la délibération sur la formation, ne constituent pas pour autant le cadre où s'expriment le mieux l'expression des

\footnotetext{
${ }^{20} \mathrm{P}$. Méhaut (1978). Formation continue, gestion du personnel et marché de la formation, Paris, Éd du CNRS, coll. «ATP ».

${ }^{21}$ N. Abboud de Mapéou (1982). «La formation continue comme nouvel outil de gestion : critique d'une idée floue », in L'emploi, enjeux économiques et sociaux, Paris, Maspéro, coll. «Textes à l'appui », p. 255-268.

${ }^{22}$ R. Sainsaulieu (1974). «L'effet de la formation dans l'entreprise », Esprit, n spécial, p. 407-428 ; R. Sainsaulieu (1978). L'identité au travail. Les effets culturels de l'organisation, Paris, Presses de la Fondation des sciences politiques ( $2^{\mathrm{e}}$ éd. 1985).

${ }^{23}$ M. Feutrié, E. Verdier (1993). «Entreprises et formations qualifiantes. Une construction sociale inachevée », Sociologie du travail, n 4, p. 469-492.

${ }^{24}$ CEREQ (2006), Regards croisés sur la formation continue en entreprise. Dix études de cas. CEREQ. Collection Net.doc. $n^{\circ} 21$.
} 
« besoins » et l'initiative individuelle, l'accès et les conditions du départ en formation étant largement subordonnés aux objectifs de l'entreprise ${ }^{25}$.

Depuis les années soixante-dix a commencé à se structurer, autour du CEREQ et de ses centres associés, un réseau de recherche sociologique et économique sur les relations entre la formation continue et l'emploi. On lui doit un ensemble de travaux empiriques, surtout à base statistique, sur les évolutions de la formation continue dans les entreprises, les branches et les régions. Le dispositif public d'information statistique s'est doté de plusieurs enquêtes, dont l'enquête «Formation continue » réalisée en 2000 et rééditée en $2006^{26}$, ainsi que l'enquête européenne CVTS coordonnée par Eurostat. Leur exploitation a permis d'enrichir la connaissance des facteurs contextuels propices à la formation, dans le secteur privé et la fonction publique ${ }^{27}$, ou encore d'identifier le caractère complémentaire ou substituable des modalités de formation (stages, formation en situation de travail, autoformation) ${ }^{28}$. Ces enquêtes permettent de mieux documenter la diversité des politiques de formation des entreprises et leur incidence sur la participation à la formation. On notera en particulier l'existence de politiques de formation, très minoritaires (11\% des entreprises) mais « vertueuses » qui offrent des opportunités de formation et d'évolution professionnelle, qui favorisent l'expression des aspirations à la formation des salariés et la formulation de leurs besoins en la matière ${ }^{29}$.

\section{Les analyses sociologiques des pratiques de formations post-scolaires}

Le regard porté par les sociologues sur les pratiques de formation est longtemps resté marqué par la théorie de la reproduction, dont l'influence s'est estompée au cours de la dernière décennie, notamment pour faire place à un vaste débat suscité par l'émergence de nouvelles formes d'organisation du travail et de la formation, mais aussi de controverses nées à propos des façons de penser la qualification ou la compétence des salariés. Ce débat et ces controverses ont contribué à valoriser des approches de type compréhensif, qui prennent en compte les significations de la formation pour les individus qui s'y engagent en tant qu'acteurs.

\subsection{De la théorie de la reproduction à celle de la segmentation}

\footnotetext{
${ }^{25}$ C. Perez et J. Vero, (2003), «L'accès à la formation en entreprise au regard des modes de gestion de la main d'œuvre », Travail et emploi, $\mathrm{n}^{\circ} 107$, p. 59-71.

${ }^{26}$ Pour une présentation synthétique des enquêtes récentes et un extrait de leurs premiers résultats factuels, voir CEREQ, (2009), Quand la formation continue, CEREQ : Marseille.

${ }^{27}$ C. Perez, (2003), « La formation continue des agents de la Fonction publique au miroir du secteur privé », Formation Emploi, n81, p. 81-97.

${ }^{28}$ S. Hanchane et M. Lambert (2003), « La variété des modes de formation : usages et enjeux », Formation Emploi, n`81.

${ }^{29}$ M. Lambert, J. Vero (2007). «Formation continue : quelles capacités d'action des salariés ? L'approche par les capacités comme grille de lecture », Formation Emploi, n98, pp. 109-141.
} 
L'impact de la théorie de la reproduction formulée par P. Bourdieu et J.-C. Passeron ${ }^{30}$ a débordé la sociologie de l'éducation pour s'appliquer au cas de la formation professionnelle, initiale et continue. Comme l'école, la formation continue véhicule des inégalités sociales et des rapports de domination qui reproduisent le capital culturel hérité et le transforment en mérites individuels justifiant la hiérarchie des positions sociales et professionnelles. Les premières études sur le public des « cours du soir » et des instituts de promotion supérieure du travail confirment ces mécanismes scolaires de reproduction sociale au sein des formations post-scolaires. Plutôt que d'offrir une "deuxième chance» à la masse des ouvriers et employés ayant quitté l'école précocement, la formation continue de type "promotion sociale » permet souvent à une fraction très limitée d'entre eux, qui disposent par leurs origines sociales d'un capital culturel plus élevé, de suivre une trajectoire de contre-mobilité, c'est-à-dire de rattraper un déclassement accidentel et temporaire en début de vie active ${ }^{31}$. Même en cas de réussite au diplôme, après un long et incertain parcours, elle ne donne accès qu'à des fonctions et des carrières inférieures à celles des diplômés d'école.

Cette approche essentiellement critique ne s'oppose pas fondamentalement à une autre perspective structurelle qui traite la formation comme une modalité de reproduction de la force de travail, fortement intégrée à la gestion du personnel et aux rapports de classe au sein de l'entreprise ${ }^{32}$. Cette analyse complexifie la question de la reproduction sociale en l'articulant avec celle des dynamiques productives et de la segmentation des marchés du travail qui en résulte. Les trajectoires des entreprises sont au moins aussi explicatives des pratiques de formation que les trajectoires individuelles. Une troisième perspective s'efforce d'articuler les deux précédentes, tout en y intégrant l'élucidation des significations différentielles que les salariés accordent à la formation selon les segments de marché du travail sur lesquels ils évoluent ${ }^{33}$. La segmentation n'est plus seulement économique et productive, elle est aussi sociale et culturelle: l'évolution des rapports des salariés à la formation est analysée en articulant les contraintes structurelles de la production et les dynamiques culturelles des biographies individuelles.

\subsection{Formations «traditionnelles» et formations « innovantes» : un débat sociologique}

Alors que la crise de l'emploi s'aggrave et que montent les questionnements sur la modernisation des entreprises, voire sur l'émergence d'un «nouveau modèle » productif, une inflexion se fait sentir au cours des années 1990 dans les recherches sur la formation. L'entreprise "flexible », engagée dans des changements techniques et organisationnels de grande ampleur, soucieuse de la qualité des produits et d'une plus forte mobilisation des

\footnotetext{
${ }^{30} \mathrm{P}$. Bourdieu, J-C. Passeron (1970). La reproduction. Éléments pour une théorie du système d'enseignement, Paris, Éd. de Minuit.

${ }^{31}$ P. Fritsch (1971). L'éducation des adultes, Paris-La Haye, Mouton ; C. de Monlibert (1968). « Promotion et reclassement. Le public des cours du soir à la recherche d'une promotion par le diplôme ", Revue française de sociologie, 9, p. 208-217 ; C. de Monlibert (1977). «L'éducation permanente et la promotion des classes moyennes », Sociologie du travail, 3, p. 246-259.

${ }^{32}$ P. Méhaut (1978). Formation continue... op. cit.

${ }^{33}$ C. Dubar (1991) La socialisation. Construction des identités sociales et professionnelles, Paris, Armand Colin (2 ${ }^{\mathrm{e}}$ éd. 1995).
} 
salariés, fait l'objet de recherches qui soulignent la portée des changements en cours. À une formation " traditionnelle » adaptative et instrumentale, caractérisée par les actions de courte durée liées à l'introduction de nouvelles installations techniques, se substituent de nouvelles formations. Celles-ci sont souvent pluri-annuelles, diplômantes ou du moins qualifiantes, élaborées en concertation avec les salariés, impliquant le personnel d'exécution, intégrées à un redéploiement d'ensemble de la stratégie de l'entreprise.

Un des aspects qui retient l'attention des sociologues est le caractère formateur reconnu, de plus en plus souvent, au travail lui-même. Il ne s'agit pas seulement, par la formation, de faciliter l'apprentissage de savoir-faire mais aussi de permettre l'acquisition et la mise en œuvre de connaissances techniques et organisationnelles, et, surtout, de «compétences sociales » : communication, capacité de travailler en équipe, prise d'initiatives... Celles-ci sont requises par les nouvelles formes d'organisation du travail qui peuvent, sous certaines conditions, devenir «qualifiantes », c'est-à-dire permettre la construction et le partage de savoirs nouveaux, y compris pour des opérateurs dont l'autonomie peut être favorisée par des liens étroits entre formation et activités de travail ${ }^{34}$. Mais en est-il réellement ainsi dans la majorité des entreprises? Cette vision, souvent trop normative et optimiste doit être relativisée. Des recherches empiriques soulignent la fragilité des expériences allant dans ce sens, dépendantes de la coopération de nombreux acteurs et vulnérables à diverses formes de dérive soit vers le modèle scolaire, soit vers l'adaptation à court terme aux besoins immédiats. Elles montrent la nécessité de prendre en compte les manières, socialement construites au cours des biographies, dont les individus vivent et expriment leur rapport à la formation ou aux innovations organisationnelles. Celles-ci ne se traduisent pas mécaniquement par une adhésion aux offres ou injonctions de la direction, surtout lorsque celles-ci n'impliquent aucune amélioration du travail ou des perspectives d'emploi à l'issue des formations ${ }^{35}$.

\subsection{L'apport des approches pratiques de formation et dynamiques identitaires}

compréhensives :

Le modèle de la « compétence ", porteur de fortes exigences cognitives et comportementales, ne rencontre souvent qu'une réaction réservée, voire craintive, de la part des salariés d'exécution qui, ayant quitté de bonne heure le système scolaire, ont appris leur métier sur le tas, et mené leur vie professionnelle à l'écart des voies de formation disponibles depuis les années soixante. L'incitation à la formation cadre mal avec leur rapport au savoir fondé sur la maîtrise du travail à partir de connaissances pratiques. Elle engendre l'inquiétude sur leur sort à l'occasion des plans sociaux qui se multiplient avec leurs compressions d'effectifs, touchant

${ }^{34}$ J.-M. Barbier, F. Berton, J.-J. Boru (1996). Situations de travail et formation, Paris, L'Harmattan.

35 J. Broda (1991). "Retours de formation: travail, rémunérations, coopérations », Formation-Emploi, n ${ }^{\circ} 34$, p. 101-115 ; C. Gadéa, F. Pottier (2003). « Salaires et trajectoires professionnelles des ingénieurs du Conservatoire national des arts et métiers : au-delà des idées reçues », Formation Emploi, n 83, p. 77-90 ; C. Gadéa, D. Trancart (2003), " Pratiques de formation continue et promotion au statut de cadre : un lien paradoxal ", Formation Emploi, n ${ }^{\circ}$ 81, pp. 99-113. 
en priorité précisément ceux qui sont perçus par la direction comme peu compétents, non motivés, incapables de faire face aux bouleversements de leur univers professionnel ${ }^{36}$.

Il peut heurter également les salariés qui s'étaient formés à un métier dans lequel ils projetaient leurs espoirs d'évolution sous la forme d'une progression hiérarchique associée à l'accroissement de qualification et d'expérience acquise par l'ancienneté. Avec la généralisation des politiques de formation centrées sur la polyvalence, les formations générales et la mobilité horizontale, ils sentent leur avenir s'assombrir d'autant plus que les formes de négociation collective de la qualification s'effritent pour laisser place à l'évaluation individuelle des compétences.

Plus conforme aux attentes des employeurs, la forme identitaire de ceux qui aspirent à prendre des responsabilités, s'élever dans la hiérarchie, et qui font preuve d'une participation active à la formation sous toutes les formes, s'est néanmoins trouvée confrontée à des obstacles de plus en plus importants. La recherche d'économies sur le coût de la maind'œuvre, le ralentissement généralisé des promotions, la réduction des lignes hiérarchiques ont touché de plein fouet cette identité d'entreprise, devenue souvent encombrante pour des entreprises qui tentaient de développer la flexibilité externe.

C'est donc avant tout pour ceux qui ont développé une "identité de réseau » et qui privilégient les formations de type universitaire, longues et diplomantes que l'évolution des politiques d'entreprises, comme des dispositions légales récentes, semble offrir le plus de chances. Valorisant l'autonomie, enclins à changer souvent d'employeur, animés par une volonté d'ascension sociale et professionnelle, ils entrent en résonance avec les discours européens sur la formation tout au long de la vie, mais au prix d'un effort constant de maintien de leur « employabilité », d'un affrontement de l'incertitude et de risques importants d'échec ${ }^{37}$

L'enseignement le plus marquant des dernières années est certainement cette montée des incertitudes, tant pour les individus concernés par la formation que pour ceux qui les décident ou les mettent en œuvre. La conjoncture des années 2000, en France, tend ainsi à jeter le doute sur la pertinence des catégories utilisées antérieurement dans le débat social (promotion sociale, innovations). De même, les catégories officielles cristallisées dans l'appareil statistique deviennent floues et inadaptées aux évolutions des politiques et des pratiques de formation. $\mathrm{Si}$, par exemple, la notion de stagiaires de la «promotion sociale » avait pu posséder une signification relativement claire lors des années soixante et soixante-dix et se prêter à des mesures fiables, elle a aujourd'hui disparu des statistiques officielles. Dans le même temps, les perturbations du marché du travail et des règles régissant la négociation entre employeurs et salariés entraînent une diversification forte des publics de la formation post-scolaire; les attentes et objectifs des candidats ne correspondent plus que de façon minoritaire aux anciennes catégories ; la formation devenant une contrainte pour les salariés qui veulent préserver leur employabilité, il devient plus difficile de savoir dans quelle mesure

\footnotetext{
${ }^{36}$ C. Dubar (1991). La socialisation, op. cit., chap. 9.

${ }^{37}$ C. Dubar, C. Gadea (1998). «Evolution de la promotion sociale et dynamique des formes identitaires », Education permanente, ${ }^{\circ} 136$, pp. 79-89.
} 
elle peut relever de l'initiative individuelle ${ }^{38}$. Les chercheurs tentent ainsi de reconstruire des catégories opératoires, s'efforçant de combiner l'analyse qualitative des sens subjectivement vécus de la formation, seule façon d'élaborer de nouvelles typologies des publics, avec des estimations quantitatives des effectifs de ces diverses catégories ${ }^{39}$.

\section{L'évaluation sociologique des inégalités face à la formation continue}

L'espoir d'une compensation des inégalités scolaires ne s'est jamais vraiment réalisé dans le cadre de la formation post-scolaire à la française qui tend à perpétuer les inégalités et à en créer de nouvelles, plus qu'elle n'en supprime. Ce n'est pas seulement dans les chances d'accès à la formation que se lisent ces inégalités, mais aussi dans ses effets en termes de promotion. La question des mécanismes sociaux producteurs d'inégalités est au cœur de nombreux travaux sociologiques qui s'efforcent souvent d'articuler l'analyse des politiques à celle des pratiques de formation.

\subsection{L'évolution des inégalités d'accès aux formations post-scolaires}

Le doute n'est guère permis : les bénéficiaires de la formation post-scolaire, en France, sont avant tout les salariés les plus qualifiés possédant une bonne formation générale et des diplômes élevés. La trajectoire sociale et scolaire joue toujours un rôle capital dans les probabilités d'entrée en formation et induit d'importants écarts entre catégories socioprofessionnelles, écarts qui se sont à peu près stabilisés depuis plus de trente ans : les chances d'aller en formation sont plus de deux fois plus fortes en moyenne pour un cadre que pour un ouvrier, elles varient du simple au triple si l'ouvrier n'a pas de diplôme et si le cadre possède un titre égal ou supérieur au niveau bac +3 . De fortes inégalités demeurent également dans les chances d'accès à la formation selon le sexe, particulièrement chez les ouvriers et employés (respectivement $30 \%$ et $44 \%$ des hommes ont suivi au moins une formation en 2006 contre $21 \%$ et $36 \%$ des femmes) mais aussi chez les cadres (62\% des hommes et $57 \%$ des femmes). La discrimination par l'âge reste elle aussi très forte, avec des taux d'accès qui varient de $51 \%$ pour les $15-29$ ans à $35 \%$ à 50 ans et plus ${ }^{40}$.

À cela s'ajoute un constat déjà ancien mais particulièrement riche d'enseignements : les facteurs structurels de l'inégalité exercent un poids croissant et nettement plus fort que les caractéristiques individuelles. La taille des entreprises, le secteur d'activité, la zone géographique, les particularités de la gestion de la main-d'œuvre ou des politiques publiques se combinent pour démultiplier les inégalités selon l'entreprise où l'on travaille, la région où l'on habite. Les différences d'accès aux formations, entre hommes et femmes, par exemple,

\footnotetext{
${ }^{38}$ M. Correia, F. Berton (2004) (dirs.). Initiative individuelle et formation. Contributions de la recherche, état des pratiques et étude bibliographique. Paris, L'Harmattan.

${ }^{39}$ A. Correia, F. Pottier (1999). «Les publics du Conservatoire national des arts et métiers. Caractéristiques socio-démographiques, parcours professionnels et raisons d'entrée en formation », L'Orientation scolaire et professionnelle, vol. $28, \mathrm{n}^{\circ} 4$.
}

${ }^{40}$ CEREQ, (2009), op.cit. 
dépendent beaucoup du mode de gestion de l'entreprise où ils travaillent. De plus, dans la mesure où le système français est bien devenu un système de formation professionnelle continue et non d'éducation permanente, il tend à exclure les individus restés à l'écart du marché du travail (femmes au foyer...) ou ceux qui en sont sortis (retraités).

Il faut cependant rappeler qu'il existe d'autres motivations et d'autres voies de formation post-scolaire que la formation professionnelle, et celles-ci ont connu un fort développement au cours des dernières années, en particulier à cause de l'essor d'internet et des technologies de la communication qui favorisent l'apprentissage individuel, aussi bien de savoirs formels (par exemple l'étude des langues avec les multimédia) que de savoirs informels (par exemple surfer sur internet et consulter les encyclopédies collaboratives) d'une infinie richesse et accessibles gratuitement ...pour qui possède un ordinateur, sait l'utiliser et peut payer un fournisseur d'accès à internet. La relative accessibilité matérielle des ressources culturelles n'empêche d'ailleurs nullement la subsistance de fortes inégalités en matière de pratiques et de « goûts » sociaux ${ }^{41}$.

\subsection{L'inégalité des effets de la formation continue}

Des inégalités supplémentaires peuvent surgir à l'issue de la formation. Une estimation, issue de l'enquête FQP de 1993, montrait déjà que les hommes déclarent plus fréquemment avoir reçu une augmentation de salaire, après formation, que les femmes les formations longues donnant plus souvent lieu à une augmentation que les autres. Des variations importantes apparaissent aussi selon le secteur, cette fois dans le sens de plus grandes probabilités d'augmentation dans les secteurs où les taux d'accès à la formation sont le moins élevés, et selon la taille, avec, là aussi, des chances d'augmentation plus sensibles dans les petites entreprises qui, par ailleurs, recourent moins fréquemment à la formation ${ }^{42}$.

Quant aux effets de la formation sur le changement de catégorie professionnelle, c'est-à-dire sur la promotion, on doit d'abord noter qu'elle s'est globalement ralentie au cours des trente dernières années, alors qu'on a pu parler à juste titre d'une explosion des effectifs de stagiaires.Dans un contexte marqué par l'instabilité de l'emploi, la formation continue accompagne les changements plus qu'elle n'assure une promotion. La probabilité d'avoir suivi une formation s'élève, selon l'enquête FQP 2003, lorsque le salarié a connu au cours des cinq années précédentes un changement de profession dans la même catégorie $(+11,8 \%)$, une promotion $(+17 \%)$, ou même un changement de poste dans le même métier $(+8,4 \%)^{43}$. Le constat le plus clair qui ressort des travaux des années 1990 est celui d'un effondrement des chances de promotion à l'issue de la formation. La plupart du temps, les salariés formés n'ont connu aucun changement dans leur situation professionnelle, ou alors il s'agit uniquement de

\footnotetext{
${ }^{41} \mathrm{Ph}$. Coulangeon (2010). " Le privilège culturel des cadres », in P. Bouffartigue, C. Gadea, S. Pochic (dirs.), Cadres, classes moyennes : vers l'éclatement? Paris, Armand Colin, p.187201.

${ }^{42}$ G. Podevin (1999). «Fomation et promotion sociale : un lien démocratique rompu ?», in Dubar et Gadea (éds.), La promotion sociale en France, op. cit., p. 143-153.

${ }^{43}$ C. Bonaïti, E. Viger (2008). «Tenter un diplôme au cours de sa carrière : peu d'occasions pour les salariés », DARES, Premières synthèses, $\mathrm{n}^{\circ} 14-3$, avril.
} 
mobilité horizontale. De même l'impact sur les salaires est peu visible ${ }^{44}$. En revanche, il se confirme que la formation avantage les salariés les plus stables et les mieux intégrés aux marchés internes des entreprises, elle permet notamment d'augmenter la rémunération des salariés appartenant aux entreprises qui payent déjà le mieux leurs salariés et elle fonctionne comme un signal en faveur de ceux qui disposaient déjà des meilleures opportunités de carrière $^{45}$.

Ces résultats ne doivent pas conduire pour autant à la démobilisation et au fatalisme sociologique peu compatible avec l'esprit qui préside à la plupart des recherches empiriques. Ils doivent, au contraire, inciter les décideurs, les formateurs et les stagiaires eux-mêmes à prendre en compte les conditions sociales de la formation, à tenir le plus grand compte des situations concrètes auxquelles les personnes sont confrontées et à donner la priorité à celles pour qui les probabilités d'accès sont les plus faibles : immigrés, ouvriers âgés, salariés peu qualifiés ${ }^{46}$. Cela requiert d'inventer des offres de formation et des organisations de travail qui leur permettent d'acquérir les qualifications requises et de mettre en œuvre des dynamiques identitaires émancipatrices. Même s'ils restent minoritaires et souvent expérimentaux, des dispositifs de formation "démocratisants » ont existé et existent encore dans le panorama des formations post-scolaires " à la française ». C'est notamment le cas de ceux qui, sur une base territoriale et avec des «pédagogies» adaptées, luttent contre les formes culturelles d'exclusion et tentent d'articuler formation et travail ${ }^{47}$.

\section{En guise de conclusion : éléments de comparaison internationale}

Les enquêtes européennes fournissent des éléments de comparaison aisément accessibles qui révèlent la disparité des situations, des pratiques et des politiques nationales de formation et permettent de situer les spécificités françaises. Il semble ainsi que la France fasse partie, avec la Suède, le Luxembourg, la République Tchèque, du groupe de pays où le taux d'entreprises formant leurs salariés et le taux d'accès des salariés sont les plus élevés. Elle se distingue également par un niveau élevé de recours aux cours et aux stages plutôt qu'à d'autres modalités de formation et par un ratio élevé de dépenses de formation continue rapportées au coût de la main d'œuvre ${ }^{48}$. Ce constat global ne doit cependant pas masquer l'existence de fortes disparités : la France se situe au-dessus de la moyenne européenne pour la proportion

${ }^{44}$ D. Fougère, D. Goux, E. Morin (2001) « Formation continue et carrières salariales. Une évaluation sur données individuelles » Annales d'Economie et de statistique, $\mathrm{n}^{\circ}$ 62, pp. 4969.

${ }^{45}$ D. Goux, E. Maurin (1997). «Les entreprises, les salariés et la formation continue », Économie et statistiques, $\mathrm{n}^{\circ}$ 306, p. 41-55 ; J.-J. Paul (1999). « La formation continue favorise-t-elle l'augmentation des salaires? », in La promotion sociale en France, op. cit., p. 154-162 ; P. Béret (2009). « Formation continue, salaires et transformation des marchés internes », Travail et emploi, 2009-1, n¹17, pp. 67-80.

${ }^{46}$ D. Gélot, C. Minni (2006). « Les immigrés accèdent moins à la formation professionnelle continue » Formation emploi, n94, pp. 93-109.

${ }^{47}$ B. Schwartz (1996). Moderniser sans exclure, Paris, La Découverte.

${ }^{48}$ Voir Quand la formation continue... op. cit. ; Checcaglini A., Marion-Vernoux I. (2008). « La formation continue dans les entreprises européennes. Premiers pas vers une homogénéisation ». Bref. $\mathrm{N}^{\circ} 251$. 
d'employés et d'ouvriers peu qualifiés n'ayant pas suivi de formation ${ }^{49}$. Un autre enseignement des comparaisons européennes concerne l'effet de la taille des entreprises : dans la mesure où, dans la plupart des pays, les grandes entreprises mènent une politique active de formation, c'est en grande partie en fonction de l'implication des petites entreprises que se creusent les différences. Les pays qui apparaissent comme offrant le plus d'accès à la formation sont ceux dans lesquels le comportement des petites entreprises se rapproche le plus de celui des grandes.

Au-delà des données quantitatives, qui mesurent des phénomènes dont la définition peut varier selon les contextes, il est important de situer la comparaison dans des cadres explicatifs susceptibles de révéler des logiques sociales - ou "sociétales »- à l'œuvre dans les divers contextes nationaux. Depuis l'étude pionnière du $\operatorname{LEST}^{50}$, cette démarche sociétale reste présente dans les approches comparatives faisant ressortir des oppositions entre les "modèles" français et allemand, français et autrichien, etc ${ }^{51}$. Mais le raisonnement en termes de "modèle » a ses limites. Il privilégie, souvent de manière excessive, le cadre national. Il simplifie les configurations d'acteurs et ne tient pas assez compte des dynamiques temporelles. Il sous-estime la convergence des politiques d'entreprises et d'institutions, et particulier dans le cadre des politiques européennes. Il néglige les significations subjectives «locales» que les individus accordent à leur parcours professionnel et, au-delà, à leur biographie personnelle. Or, pour comprendre les pratiques de formation, il faut connaître les cadres de référence, les attitudes et les visions du monde des adultes impliqués dans des dynamiques qui mettent en jeu leur « projet de vie ».

C'est en cela que le cadre national ne s'avère pas toujours le plus pertinent: il faut tenir le plus grand compte des contextes locaux comme des dynamiques globales. C'est en s'efforçant d'articuler ces différents niveaux d'analyse que les sociologues ont le plus de chances de produire des résultats comparatifs permettant de relier «les structures institutionnelles et les expériences vécues ${ }^{52} »$.

\footnotetext{
${ }^{49}$ Boateng S. K. « Significant adult differences in adult learning », Eurostat, Statistics and Focus, Population and social conditions, $\mathrm{n}^{\circ} 44$.

${ }^{50}$ M. Maurice, F. Sellier, J.-J. Silvestre (1982). Politiques d'éducation et organisation industrielle en France et en Allemagne, Paris, PUF, coll. " Sociologies ».

${ }^{51}$ J.-P. Géhin, P. Méhaut (1993). Apprentissage ou formation continue? Stratégies éducatives des entreprises en Allemagne et en France, Paris, L'Harmattan ; N. Normand (2001) " La formation professionnelle continue et les enjeux de l'évaluation" \& "De la norme au territoire : la construction locale d'un dispositif d'évaluation de la Formation Professionnelle Van Haecht A. (2001) «Evaluation et comparaison des politiques et systèmes de formation professionnelle continue en Europe », Revue de l'Institut de Sociologie, n¹-4, Ed. Université Libre de Bruxelles; Machado L. (2008). «Les formes de la formation continue en entreprise: une conséquence des acceptions sociétales? Comparaison de l'Autriche et de la France », Revue européenne de formation professionnelle $\mathrm{n}^{\circ} 44$, pp. 35-52.
}

${ }^{52}$ C. Dubar, C. Gadéa, C. Rolle (1998). «Formation continue et promotion sociale : une comparaison européenne », in M. Lallement, J. Spurk (éds.), Stratégies de comparaisons internationales, Paris, CNRS Éditions. 
Quoi qu'il en soit, les comparaisons, notamment européennes, commencent à se développer dans le champ de la formation, comme partout ailleurs. Les recherches permettent de prendre plus clairement conscience que le terme "formation » n'a pas le même sens dans tous les pays européens et que ses liens avec le travail, l'emploi, la mobilité ne sont pas pensés partout de la même manière ${ }^{53}$. Sans doute la capacité à connaître ces liens et à agir sur eux représente-t-elle l'une des clefs de la convergence recherchée par les politiques européennes. Ces dernières doivent s'appuyer sur les résultats et les pistes de réflexion qui ressortent des recherches sociologiques. Celles-ci-font constamment ressortir combien l'idéal démocratique qui inspirait Condorcet demeure loin d'être atteint, mais aussi combien les efforts réels consentis par les états et par la Communauté européenne se heurtent à des logiques complexes. La dégradation de la conjoncture économique depuis 2008 se traduit par un ralentissement de la politique de formation des entreprises, et risque d'aggraver le scepticisme de certains salariés déçus de voir que la formation continue ne permet pas de satisfaire leurs attentes de promotion ou d'amélioration de leur rémunération. Mais des travaux récents suggèrent aussi que l'appétence des salariés les moins qualifiés -ceux qui ont le plus besoin de formation- pourrait augmenter sensiblement s'ils disposaient d'une meilleure visibilité sur ce que représente concrètement pour eux l'engagement dans une opération de formation continue et sur ce qu'il est possible d'en attendre ${ }^{54}$. La recherche sociologique permet non seulement de prendre conscience des failles, des lacunes, des effets inattendus des politiques de formation lancées par les entreprises et les pouvoirs publics, mais aussi du sens que les individus donnent à la formation, des conditions dans lesquelles leurs chances d'accès pourraient s'élever, et des enjeux sociétaux dans lesquels elle s'inscrit. Si elle porte la trace des tensions et injustices qui traversent le monde du travail, la formation post-scolaire constitue également de longue date un «laboratoire d'expérimentations sociales et politiques $^{55} »$, un des lieux essentiels du dialogue entre partenaires sociaux.

\section{Lectures conseillées}

BLASCO S., LE J., MONSO O. (2009) «Formation continue en entreprise et promotion sociale : mythe ou réalité ? Insee Références, Collection Formations et Emploi, Edition 2009 .

BRUCY G., CAILLAUD P., QUENSON E., TANGUY L., (2007). Former pour réformer. Retour sur la formation permanente (1945-2004), La découverte, Collection « Recherches ».

Collectif (2009) Quand la formation continue. Repères sur les pratiques de formation des employeurs et des salariés, Marseille, Céreq.

53 A. Jobert, C. Marry, L. Tanguy (1995). Éducation et travail en Grande-Bretagne, Allemagne et Italie, Paris, Armand Colin; Lallement M. (2000) «La comparaison des systèmes de formation continue. Trois remarques en guise de commentaire », Cahiers $d u$ Lasmas, 01-1.

${ }^{54}$ C. Fournier (2004) « Aux origines de l'inégale appétence des salariés pour la formation », Bref Ceréq n²09.

${ }^{55}$ L. Tanguy (2007) « De la négociation entre interlocuteurs sociaux au dialogue social entre partenaires », in Brucy et al., Former pour réformer, op. cit. 
DUBAR C., GADEA C., PEREZ C., 2011, "Sociologie de la formation post-scolaire" in Traité des sciences et techniques de la formation, P. Carré et P. Caspar (dir.), DUNOD, pp.125-142

DUBAR C. (2004) La formation professionnelle continue. Paris, La Découverte.

FOURNIER C., avec la collaboration de SIGOT J.-C. (2009). "Concilier vie familiale et formation continue : une affaire de femmes ». Bref Céreq ${ }^{\circ} 262$.

LAOT F., de LESCURE E. (2008). Pour une histoire de la formation. Paris, L'Harmattan.

MORVAN Yves (2006) (dir) La formation tout au long de la vie : nouvelles questions, nouvelles perspectives. Rennes, Presses universitaires de Rennes.

NEYRAT Frédéric 2007 La validation des acquis de l'expérience. La reconnaissance d'un nouveau droit. Les éditions du Croquant. 